\title{
Mycetomas: The Experience of the Dermatology Unit of the University of Milan
}

\author{
Gianluca Nazzaro Stefano Veraldi \\ Department of Pathophysiology and Transplantation, Università degli Studi di Milano, \\ I.R.C.C.S. Foundation, Cà Granda Ospedale Maggiore Policlinico, Milan, Italy
}

\section{Keywords}

Mycetoma · Eumycetoma · Epidemiology · Tropical disease · Dermatopathology ·

Actinomycetoma

In the period from 1984 to 2016, we observed 16 patients in whom a clinical diagnosis of mycetoma was made. In 8 patients ( 6 males and 2 females, with an age ranging from 6 to 59 years [mean age: 37.1 years]), this diagnosis was confirmed by histopathological, mycological, and bacteriological examinations. In 2 patients, it was possible to perform polymerase chain reaction (PCR). The histopathological picture was superimposable in all patients. It showed suppurative granulomas surrounding characteristic grains in the deep dermis and subcutis (Fig. 1). In particular, granulomas were composed of neutrophils, histiocytes, and lymphocytes. In actinomycetomas, thin filamentous bacteria were present (Fig. 2); in eumycetomas, eosinophilic structures containing hyphae and spores were observed (Fig. 3). Complete patients' characteristics and etiological agents are reported in Table 1. In 2 cases, the infection was acquired in India, in 2 in Ethiopia, an in 1 case each in Senegal, Tanzania, Honduras, and Italy. Etiological agents were identified in 6 patients: Actinomadura madurae and Madurella mycetomatis in 2 patients; Aspergillus nidulans and Phialophora verrucosa in 1 patient; in 2 cases the infection was caused by Acremonium spp. and Fusarium spp. In 5 patients, mycetoma involved the foot; the buttocks, thigh, and ankle were involved in 1 patient each.

Mycetomas are chronic and deep infections of the skin caused by bacteria (actinomycotic mycetomas or actinomycetomas) [1,2] or fungi (eumycotic mycetomas or eumycetomas) [1, 3]. The latter are observed mainly in Sub-Saharan Africa, whereas actinomycotic mycetomas 
Fig. 1. A deep dermal suppurative granuloma. HE. $\times 100$.

Fig. 2. Mixed infiltrate composed of histiocytes, giant cells, and lymphocytes surrounding bacteria in a case of actinomycetoma. HE. $\times 400$.

Fig. 3. A case of eumycetoma: inflammatory cells surrounding eosinophilic structures containing hyphae and spores. HE. $\times 200$.

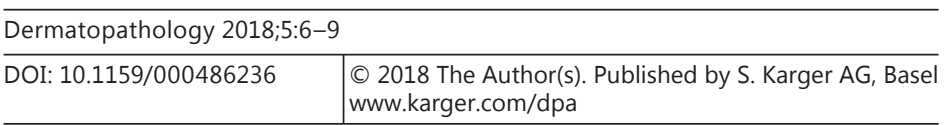

Nazzaro and Veraldi: Mycetomas: The Experience of the Dermatology Unit of the University of Milan
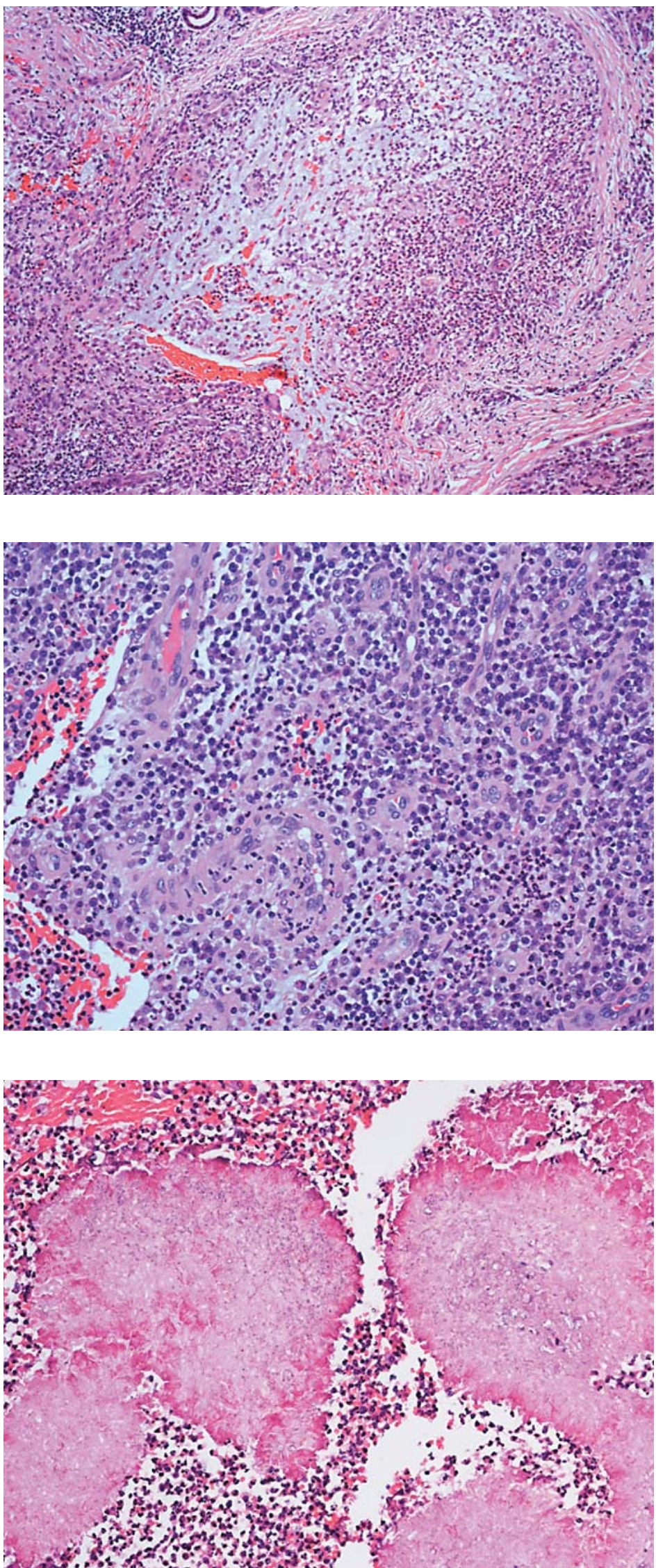
Nazzaro and Veraldi: Mycetomas: The Experience of the Dermatology Unit of the University of Milan

Table 1. Patients' characteristics and etiological agents

\begin{tabular}{|c|c|c|c|}
\hline Sex & Age, years & Location & Etiological agent \\
\hline $\mathrm{F}$ & 36 & dorsum of the left foot & Madurella mycetomatis \\
\hline $\mathrm{F}$ & 47 & plantar surface of the left foot & Madurella mycetomatis \\
\hline M & 43 & left ankle & Actinomadura madurae \\
\hline M & 39 & plantar surface of the right foot & Phialophora verrucosa \\
\hline M & 6 & right thigh & Fusarium spp. \\
\hline M & 33 & left buttock & Acremonium spp. \\
\hline M & 59 & left foot & Aspergillus nidulans \\
\hline M & 34 & plantar surface of the right foot & Actinomadura madurae \\
\hline
\end{tabular}

occur in Central and South America. Eumycotic mycetomas are caused by phaeohyphomycetes (Exophiala spp., Madurella spp.) or hialohyphomycetes (Acremonium spp., Aspergillus spp., Fusarium spp., Pseudallescheria spp.) [4]. Etiological agents penetrate the skin through breaks caused by thorns, shrubs, and wood splinters: mycetoma is considered an occupational disease in farmers and lumberjacks. Latency time may be very long (months, years). Mycetomas are characterized clinically by the slow appearance of mildly inflammatory papules, nodules, and plaques. With time, abscesses, fistulae, and ulcers appear, with a serouspurulent discharge containing "grains" (i.e., colonies of the involved species). White or yellow grains are typical of $A$. madurae, A. nidulans [4], and Pseudallescheria boydii; red grains are typical of Actinomadura pelletieri; black grains of Aspergillus terreus, Exophiala jeanselmei, and M. mycetomatis. Superinfections by Staphylococcus aureus, gram-negative bacteria, and anaerobes are frequent. Mycetomas are usually located on the legs, ankles, and feet ("Madura foot"); the buttocks and thighs may be also involved. The involvement of muscles, aponeuroses, ligaments, tendons, joints, and bones is rather common. Local symptoms are usually mild, except for difficulty walking. The clinical course is chronic-relapsing [1]. The diagnosis of mycetoma is based on medical history, clinical picture, histopathological examination, mycological and bacteriological cultures, and PCR. The use of X-rays, ultrasonography, and computed tomography scan is necessary in order to evaluate the involvement of deep tissues. The therapy of eumycotic mycetomas is difficult and very long [5]. Several systemic antimycotics, such as fluconazole, itraconazole, ketoconazole, posaconazole, voriconazole, and terbinafine, have been used. The association itraconazole-terbinafine seems to be more effective than itraconazole alone and terbinafine alone. Isavuconazole is a promising drug. Surgery is necessary in most of the patients.

\section{Statement of Ethics}

The authors have no ethical conflict to disclose.

\section{Disclosure Statement}

The authors have no conflicts of interest to disclose. 
Nazzaro and Veraldi: Mycetomas: The Experience of the Dermatology Unit of the University of Milan

\section{References}

1 Nenoff P, van de Sande WW, Fahal AH, Reinel D, Schöfer H: Eumycetoma and actinomycetoma - an update on causative agents, epidemiology, pathogenesis, diagnostics and therapy. J Eur Acad Dermatol Venereol 2015; 29:1873-1883.

-2 Arenas R, Fernandez Martinez RF, Torres-Guerrero E, Garcia C: Actinomycetoma: an update on diagnosis and treatment. Cutis 2017;99:E11-E15.

-3 Al-Hatmi AM, Bonifaz A, Tirado-Sánchez A, Meis JF, de Hoog GS, Ahmed SA: Fusarium species causing eumycetoma: report of two cases and comprehensive review of the literature. Mycoses 2017;60:204-212.

$\checkmark 4$ Veraldi S, Grancini A, Venegoni L, Merlo V, Guanziroli E, Menicanti C, Nazzaro G, Tortorano A: Mycetoma caused by Aspergillus nidulans. Acta Derm Venereol 2016;96:118-119.

5 Ameen M: Managing mycetomas. Trop Doctor 2009;39:66-68. 